\title{
Assessment of the Seismic Non-Linear Behavior of Ductile Wall Structures Due to Synthetic Earthquakes
}

\author{
PHILIPPE SCHWAB ${ }^{1}$ and PIERINO LESTUZZI ${ }^{1, *}$ \\ ${ }^{1}$ IS-Structural Engineering Institute, EPFL-Ecole Polytechnique Fédérale de Lausanne \\ ${ }^{*}$ Corresponding author: \\ EPFL ENAC IS IMAC, Bâtiment GC, Station 18, CH-1015 Lausanne, Switzerland. \\ Phone: +41 2169363 62; fax: +41 2169347 48; E-mail address: pierino.lestuzzi@epfl.ch
}

\begin{abstract}
In this paper different methods for generating synthetic earthquakes are compared in terms of related nonlinear seismic response of ductile structures. The objective of the investigation is to formulate recommendations for the use of synthetic earthquakes for reliable seismic analysis. The comparison is focused on the accuracy of the reproduction of the characteristics of the structural non-linear response due to recorded earthquakes. First the investigations are carried out for non-linear single-degree-of-freedom systems. Later the results are validated for a set of realistic buildings modelled as multi-degree-of-freedom systems.

Various options of the classical stationary simulation procedure of SIMQKE and a non-stationary simulation procedure proposed by Sabetta \& Pugliese are examined and compared. The adopted methodology uses a set of recorded earthquakes as a reference. Hundred synthetic accelerograms are generated for each examined simulation option with the condition that the related elastic responses are similar to those of the reference set. The non-linear single-degree-of-freedom systems are defined using six recognized hysteretic models and four levels of increasing non-linearity. The non-linear responses computed for the reference set and the studied simulation options are then statistically compared in terms of displacement ductility and energy.

The results show that the implementation of the classical stationary procedure always leads to a significant underestimation of the ductility demand and a significant overestimation of the energy demand. By contrast, non-stationary time histories produce much better results. The results with the multi-degree-of-freedom systems are shown to confirm these conclusions.
\end{abstract}

KEYWORDS: ductility demand, hysteretic energy, non-linear structural response, non-stationary simulation, recorded earthquake, seismic analysis, stationary simulation, synthetic earthquake.

\section{Introduction}

This paper presents the main results gained during the master thesis performed by the first author at the Swiss Federal Institute of Technology in Lausanne (EPFL). The complete description of this work may be found in Schwab (2002). The research project addresses nonlinear time history analysis in the context of structural seismic evaluation. Specifically, it aims to bring some insight to the following question: what is the impact of the generation method for synthetic earthquakes on the non-linear seismic response of ductile structures such as capacity designed reinforced concrete structural walls?

\subsection{Context}

In large number of seismic designs, evaluations and upgrade of structures, it is often useful to complement simplified methods using elastic response spectrum and strength reduction factors with a non-linear dynamic analysis. This is especially true for seismic evaluations of existing structures where it is desirable to approach as close as possible the real behavior of the 
structure. Moreover, the importance of non-linear time history analysis for design and retrofit will increase in the future with the development of more complex models and analytical tools and as these tools become available to design engineers. Modern codes and guidelines foresee this possibility and contain some recommendations for its utilisation.

Non-linear time history dynamic analysis needs a reliable model for structural behavior and an adequate definition of the seismic input. The investigations reported in this paper deal with the second issue, the seismic input. The choice of the earthquake excitation is an important parameter in the seismic structural response, specifically in case of non-linear behavior of the structure. Both recorded and synthetic earthquakes may be used for this purpose. Generally, recorded earthquakes should be preferred since they feature actual events. However, seismic analysis should mostly be performed according to the code requirements. Even if a growing number of recorded earthquakes is available in an improved database (Smit et al., 2000), it is generally difficult to find one event which satisfactorily fits the design spectrum, even in a limited frequency range. Moreover, in many regions with moderate seismicity, such as in Switzerland, no strong motion has been recorded since the time when measurements have been possible. In the engineering community, it is generally believed that synthetic time histories covering the whole frequency range of a design spectrum represent a conservative input. However, this is not necessarily true for non-linear systems. Furthermore, in case of non-linear behavior, large variability is a well known characteristic of the seismic response. In order to take this variability into account, seismic analysis should be repeated several times with different accelerograms. For these reasons, synthetic earthquakes are commonly used because of their advantage of generating large number of time histories compatible with a prescribed design spectrum.

Even if modern codes and guidelines give the number of accelerograms to be used for nonlinear time history seismic analysis (for instance Eurocode 8 (2004) and FEMA 273 (1997) require a minimum of 3 accelerograms if the most adverse of the response results is to be used, or at least 7 , if response results are to be averaged), no guidelines are given to the engineer to determine how to choose the accelerograms that are appropriate to a given design or upgrade situation. Some studies investigated this basic question for recorded earthquakes (Elenas, 2002; Lestuzzi et al., 2004), but no systematic investigations were performed for synthetic earthquakes.

Various methods have been proposed for generating synthetic ground motions which are quite different in both complexity and objective. However, the preoccupations of seismologists and structural engineers does not necessarily coincide in a ground motion simulation. While the priority of the structural engineer is a realistic simulation of dynamic response of the structure, the seismologist attaches more importance to the physical characteristics. The paper adopts the structural engineering point of view, which may significantly diverge from the seismological point of view. From structural engineering point of view, generated earthquakes may be considered as reliable if they provide similar seismic response as recorded earthquakes. Although reproduction of all the physical features of a real earthquakes is desirable, it is not a realistic goal in structural engineering due to the lack of knowledge of important parameters. As a consequence, even though there exist more sophisticated generation methods to reproduce all the characteristics of recorded earthquakes, the investigations concentrate on generation methods commonly used in structural engineering. Therefore, the investigated methods may appear very simple for seismologists.

The stationary generation method examined, is the well known classical simulation procedure of SIMQKE (Gasparini and Vanmarcke, 1976). This is the commonly used method of generating synthetic earthquakes, which are compatible with a prescribed design spectrum. This method is based on random vibration theory and is therefore a stationary simulation. In this method, the matching of the target spectrum may be improved by the means of an iterative process. The influence of this option is also considered in the investigations. The non-stationary simulation procedure investigated in this paper is the one proposed by Sabetta and Pugliese (1996). This is an empirical method fitted to the recorded accelerograms of Italian earthquakes. 
Therefore, the method of Sabetta and Pugliese is supposed to provide a realistic simulation of recorded earthquakes. This type of synthetic earthquake is generated using only three parameters (i.e. magnitude, epicentral distance and soil condition). Note that this method was not intended to generate spectrum-compatible earthquakes.

\subsection{Methodology}

Figure 1 illustrates the methodology used in the investigations. It is based on the selection of a set of recorded earthquakes to provide realistic seismic responses as a reference. In other words, the related acceleration response spectra and seismic non-linear responses are used as a basis for the comparison of the simulation methods.

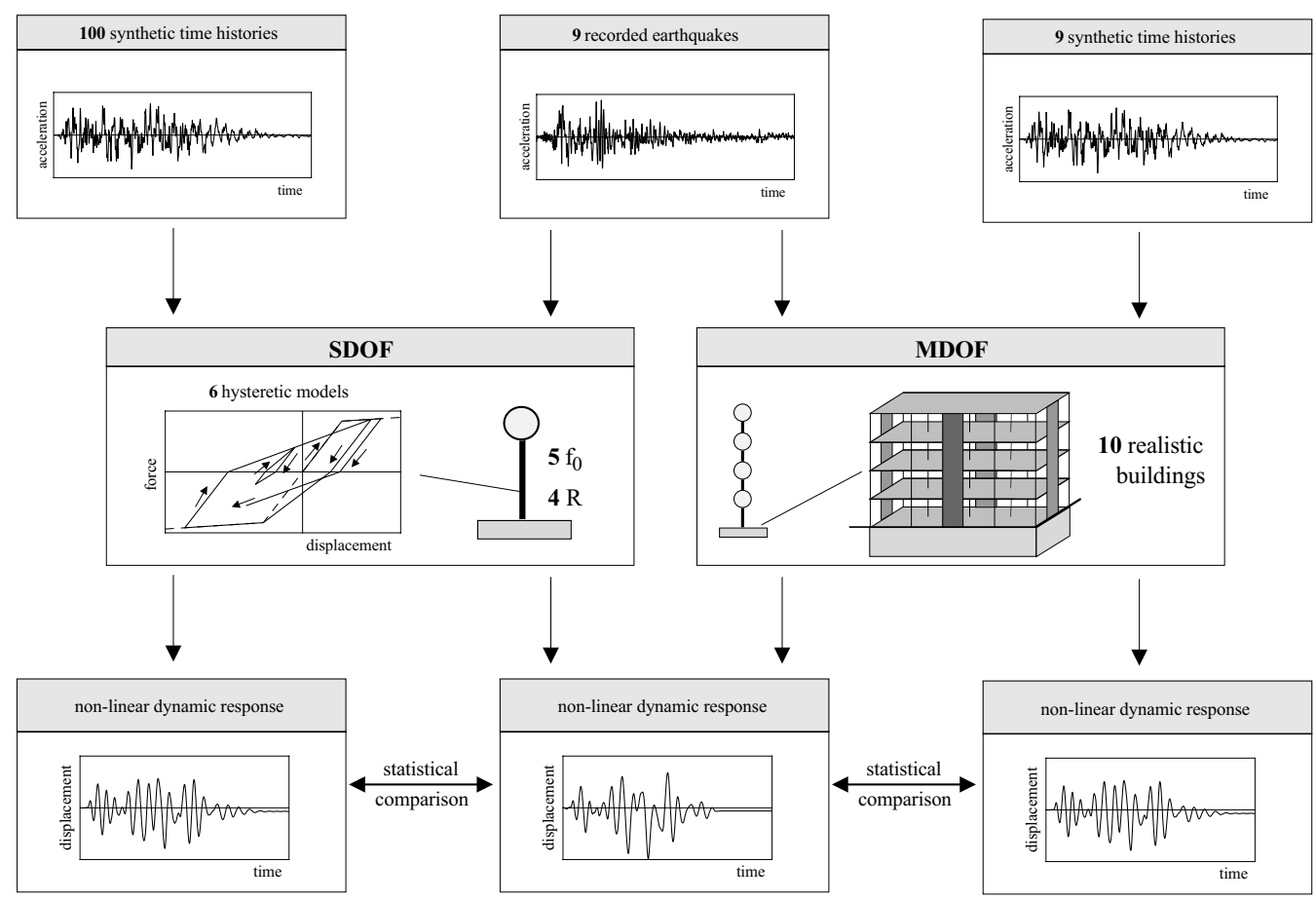

Figure 1: Schematic description of the followed methodology.

First, a set of nine recorded earthquakes featuring similar acceleration response spectra is selected. Other characteristics such as peak ground acceleration (PGA), duration, etc. are not considered in the selection. Based on the acceleration response spectra of the set of recorded earthquakes, a study spectrum is defined. The study spectrum is used as a target for the generation of some of the synthetic earthquakes.

Hundred synthetic time histories are generated for each of the five examined types of generation methods under the constraint that their elastic responses are compatible to those observed for the reference set of recorded earthquakes. The structures that undergo the seismic excitations are first and foremost modelled as non-linear single-degree-of-freedom systems (SDOF) using six recognized hysteretic models. The comparison is performed for five initial natural frequencies representing the typical natural frequencies of buildings. The ductility demand and the energy demand are considered. The comparisons are not only focused on the mean values but also on the standard deviations representing the variability of the seismic response. 
As some discrepancies between the characteristics of the seismic non-linear behavior of SDOF and the ones of multi-degree-of-freedom systems (MDOF) were already reported (Nassar and Krawinkler, 1991), the investigations are extended to MDOF. The motivation behind this second part of the investigations is to test if the results gained with SDOF hold true for realistic buildings. For this purpose, the buildings are modelled as MDOF. The idea is to perform the computations in the same manner as a design engineer. Especially, only a few time histories, instead of hundred, are used to study the variability of the non-linear responses which are, in this case, described by the displacement demand on the top story.

\section{Ground motions}

For the purpose of comparison, it is important that both the recorded and the synthetic earthquakes produce similar elastic responses. Then, it is possible to highlight the differences that arise specifically in the non-linear range.

\subsection{Recorded earthquakes}

Let us stress that an engineer's and not a seismologist's point of view is adopted in the investigations. As a consequence, earthquakes triggered in different geological conditions may be incorporated in the same reference set. This choice is justified by the fact that the main objective is to conduct a statistical study of the non-linear response of structures undergoing recorded earthquakes.

Based on the European Strong Motion Database (Smit et al., 2000) nine recordings, which produce almost the same elastic response spectra, are chosen. Their main characteristics are listed in Table 1. The magnitudes range from 5.3 to 7.8 and the peak ground accelerations (PGA) range from 1.13 to $1.72 \mathrm{~m} / \mathrm{s}^{2}$.

Table 1: Main characteristics of the nine recordings composing the reference set.

\begin{tabular}{|c|c|c|c|c|c|}
\hline Earthquake & Date & Station & Magnitude & Component & PGA [m/s ${ }^{2}$ ] \\
\hline Killini & 16.10 .88 & Zakinthos & 5.7 & SE & 1.48 \\
\hline Komillion & 25.02 .94 & Lefkada & 5.4 & Long. & 1.72 \\
\hline Aftershock Spitak & 07.12 .88 & Gukasian & 5.8 & NS & 1.45 \\
\hline Thessalonika & 20.06 .78 & City Hotel & 6.4 & NS & 1.37 \\
\hline Volvi & 04.07 .78 & City Hotel & 5.1 & NS & 1.13 \\
\hline Killini & 16.10 .88 & Amaliada & 5.7 & EW & 1.53 \\
\hline Kocaeli & 17.08 .99 & Goynuk & 7.8 & NS & 1.35 \\
\hline Preveza & 10.03 .81 & OTE Building & 5.3 & NS & 1.40 \\
\hline Pulumur & 15.03 .92 & Erzincan & 5.8 & NS & 1.13 \\
\hline
\end{tabular}

The acceleration time histories of the selected nine recordings are plotted in Figures 2, 3 and 4. Their corresponding response spectra are plotted in Figure 5.

\subsection{Synthetic stationary earthquakes}

The commonly used method of generating synthetic earthquakes, which are compatible with a prescribed design spectrum, is based on random vibration theory. The generated synthetic earthquakes correspond only to a pure mathematical description and is represented as a limited duration segment of a stationary random function. Even if they are commonly used for 

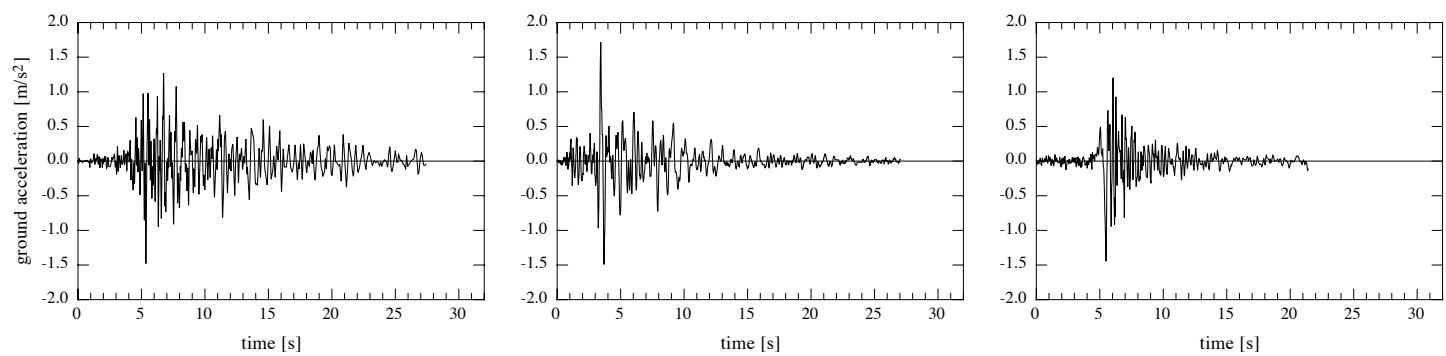

Figure 2: Selected recordings: Killini (Zakinthos), Komillion and Aftershock Spitak (from left to right).
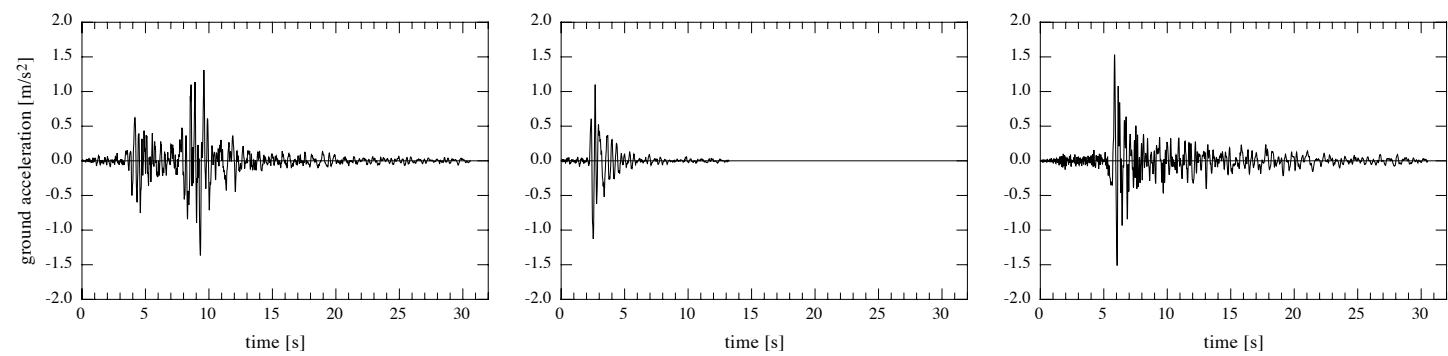

Figure 3: Selected recordings: Thessalonika, Volvi and Killini (Amaliada) (from left to right).
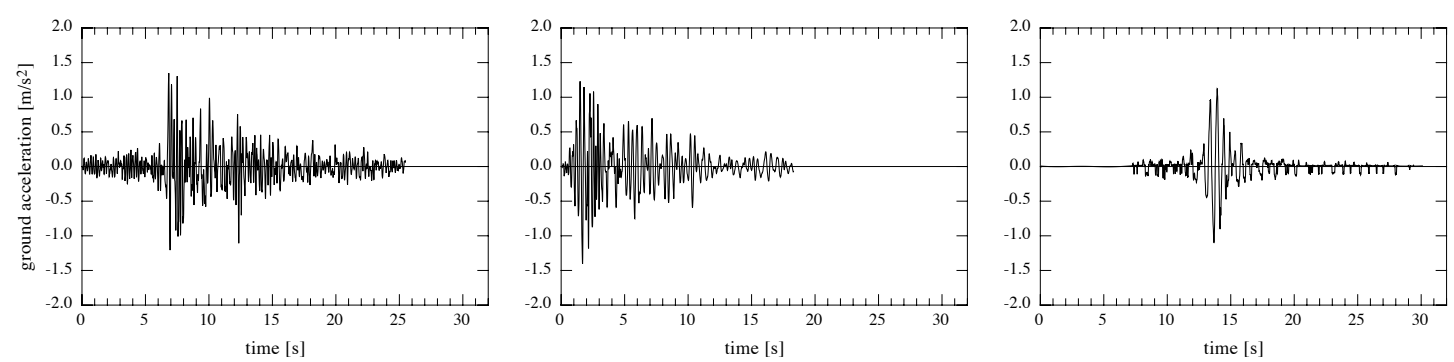

Figure 4: Selected recordings: Kocaeli, Preveza and Pulumur (from left to right).

determining dynamic structural response, they do not exhibit any physical characteristics of real recorded earthquakes. Consequently, they may only simulate the frequency content of a real earthquake during the strong motion phase. Time history characteristics of synthetic earthquakes diverge from those of recorded earthquakes. Note that this procedure was first developed to enable dynamic elastic calculations (Gasparini and Vanmarcke, 1976). This method has been implemented in the well known computer program SIMQKE (Gasparini and Vanmarcke, 1976). The program simulates a synthetic earthquake ground motion by superposition of sinusoidal waves. The ground acceleration time history corresponds to a stationary Fourier series, whose amplitudes are defined by a relationship that ties them to the target response spectrum. This relationship actually represents the core of the SIMQKE method. It is only the randomly chosen phases that differ between two earthquakes targeting the same spectrum. The signal that varies between $\pm \mathrm{PGA}$ is multiplied by an envelope that smooths the beginning and the end of the event to simulate the non-stationary transient feature. A compound envelope (Gasparini and Vanmarcke, 1976) is used throughout this study.

As the relationship between the Fourier amplitudes and the target spectral acceleration is only valid in terms of the mean values, some divergences between the acceleration response 
spectrum and the target spectrum appear. Two options are available for reducing such discrepancies:

(1) Use the original SIMQKE iterative process that improves the matching of the elastic response spectrum of the generated earthquake with the target spectrum by adapting the amplitudes. The use of such iterations is very widespread.

(2) Impose a tolerance zone around the target that clearly shows the synthetic earthquakes that should be rejected because of very different elastic responses. As no iterations are used, the search for events is more time consuming than option (1).

The impact of both the options on the non-linear structural responses is compared in the investigations.

The major input parameter required by the SIMQKE procedure is the target response spectrum. This target has to be chosen such that it enables the generation of time histories that produce elastic responses similar to the average of the response spectra computed for the recorded earthquakes. During the investigations, this condition is first fulfilled by using the average response spectrum as target spectrum. Later, an alternative method is investigated by defining a study spectrum, which approaches the mean spectral response obtained for the reference set, but with a shape close to a design spectrum. Indeed, it is based on a Eurocode (soil condition A) spectrum with the exception that a single curve at power 1.42 models the branch in the lower frequencies. As mentioned above, it may be necessary to define the extent to which the generated earthquakes qualify as giving similar elastic responses. Therefore, an upper and a lower limit are defined at $\pm 25 \%$ of the study spectrum.

The acceleration response spectrum of the nine selected recordings are plotted in the left hand side of Figure 5 together with the mean acceleration response spectrum (dash dotted line). The study spectrum which is defined to represent the mean spectrum in the low frequency range is plotted (solid) in the right hand side of Figure 5. The limits which are used for selecting or rejecting the generated earthquakes are also plotted (dotted).
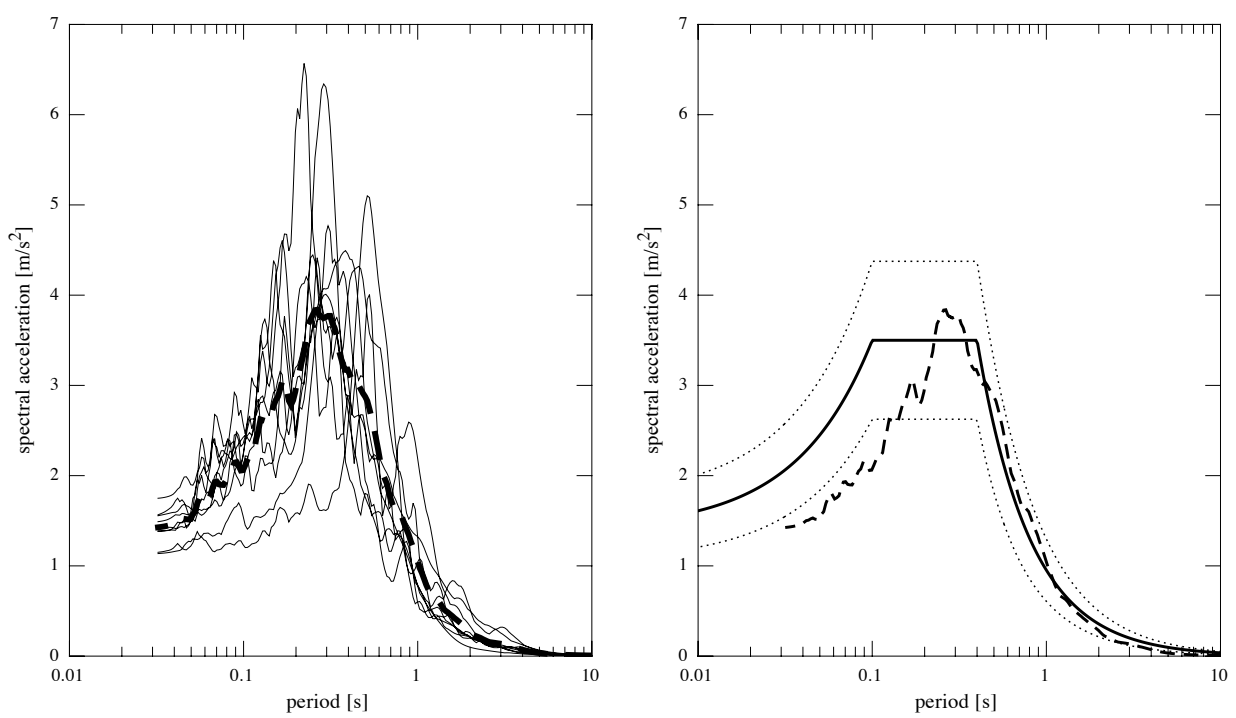

Figure 5: Left, acceleration response spectra of the nine selected recorded earthquakes along with their average (dislocated line). The study spectrum (solid line) which is used as target spectrum for the generation is shown on the right as well as the limits (dotted lines) that define to what extent the elastic response of a synthetic earthquake is similar to the average response of the recorded ones. 


\subsection{Synthetic non-stationary earthquakes}

Apart from displaying different characteristics regarding elastic response spectra, recorded and stationary synthetic earthquakes also diverge on another major aspect: the non-stationarity. Since recorded earthquakes are non-stationary signals, the Fourier amplitudes used to build the signal varies with time.

Hence, an empirical method developed by Sabetta and Pugliese (1996) that enables the generation of non-stationary earthquakes is chosen as a simulation tool for the investigations. This is an empirical generation process fitted according to 99 records of 17 italian earthquakes, whose magnitudes range from 4.6 to 6.8. The principal advantage of the method consists in correlating the simulation parameters with three input parameters: earthquake magnitude (M), source distance $(\mathrm{R})$ and local site conditions (three possible conditions related to the thickness of the alluvium deposit). Consequently, the simulated time histories fit recorded accelerograms in terms of several ground motion characteristics such as peak acceleration, peak velocity, Fourier spectra and response spectra. Even if the procedure does not include a process to match design spectrum, it is possible to generate ground motions, which in terms of mean values are compatible to a target spectrum for a limited band of frequencies, by adapting the three input parameters (magnitude, source distance, soil condition). As it may be seen in Figure 7 (right), a magnitude of $\mathrm{M}=6.3$, an epicentral distance of $\mathrm{R}=27 \mathrm{~km}$ and shallow alluvium soil conditions lead to a good compatibility in terms of mean spectral values (dash) for the study spectrum (dash dotted line).

\subsection{Studied simulation options}

Table 2 summarizes the applied synthetic earthquake generation methods. The first simulation option corresponds to the tool a design engineer is most likely to use, namely applying the SIMQKE procedure for a code shaped target spectrum and allowing up to 10 iterations to improve the match with the elastic response spectrum. The next three options are still based on SIMQKE but differ from the first one by either using another kind of target or permitting no iterations or both. The last simulation option examined is based on the Sabetta \& Pugliese method. Figure 6 illustrates each simulation option by a time history (left) and response spectra plot (right). Figure 7 gives a more general view in which the average spectra corresponding to the recorded set of earthquakes ( 9 events, dotted line), the four stationary simulation options (100 events each) and the single non-stationary simulation (100 events, dashed line) are shown.

Table 2: The five simulation options used for the investigations.

\begin{tabular}{|c|c|c|c|}
\hline Generation & Simulation option & Target & Iteration allowed \\
\hline 1 & original SIMQKE & study spectrum & 10 \\
\hline 2 & SIMQKE without iterations & study spectrum & 0 \\
\hline 3 & SIMQKE, recorded & average spectra of the recorded events & 10 \\
\hline 4 & SIMQKE, recorded, without iterations & average spectra of the recorded events & 0 \\
\hline 5 & Sabetta \& Pugliese & average spectra of the recorded events & non stationary \\
\hline
\end{tabular}

\section{Investigations with SDOF}

\subsection{Definition and hysteretic models}

The non-linear SDOF is defined by its initial fundamental frequency $\mathrm{f}_{0}$, its strength reduction factor $\mathrm{R}$ and the hysteretic model according to which the structure behaves in the non-linear 

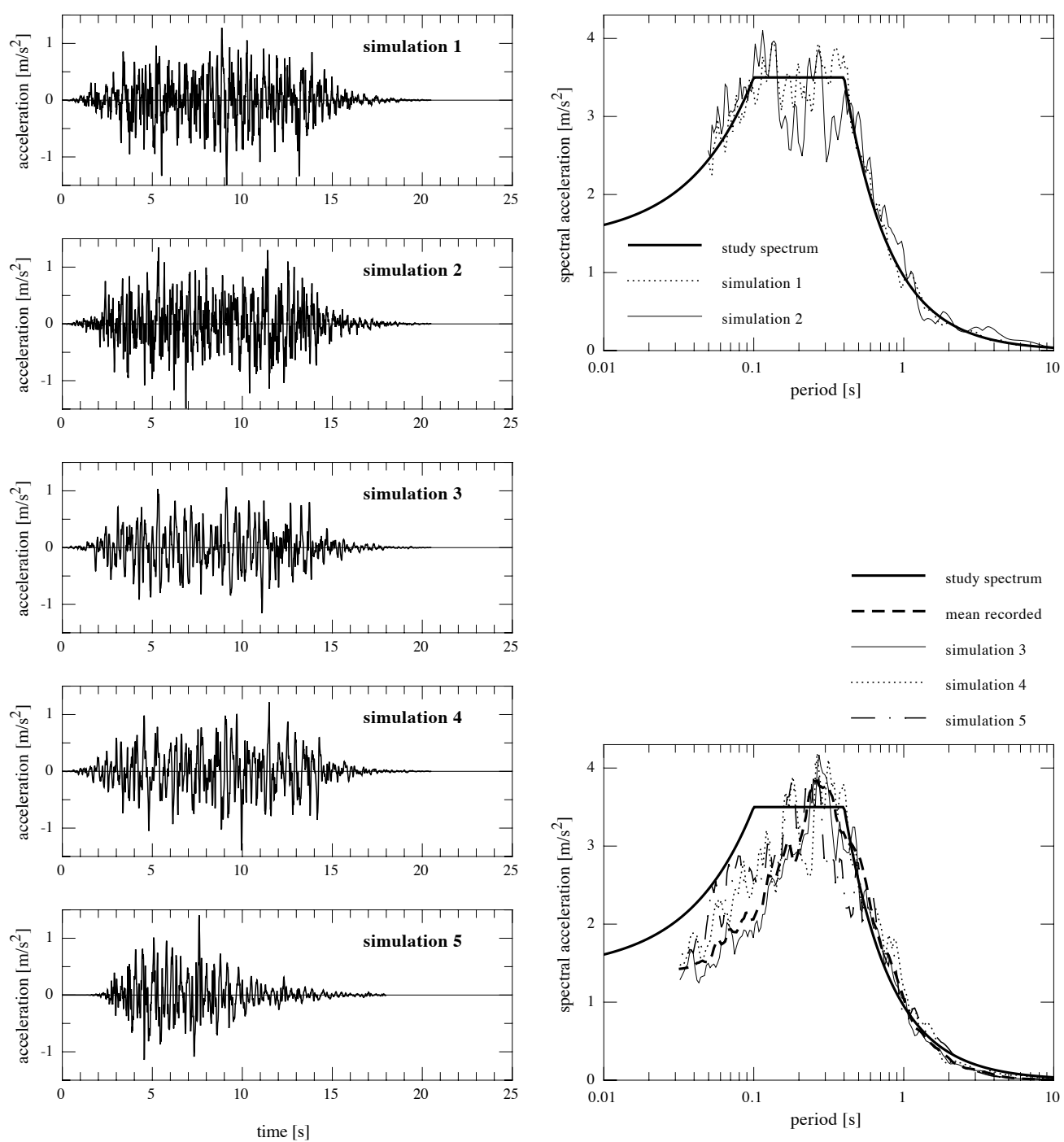

Figure 6: Typical time histories for simulation option 1 to 5 (left) and (right) corresponding elastic response spectra in comparison with the study spectrum (solid line).
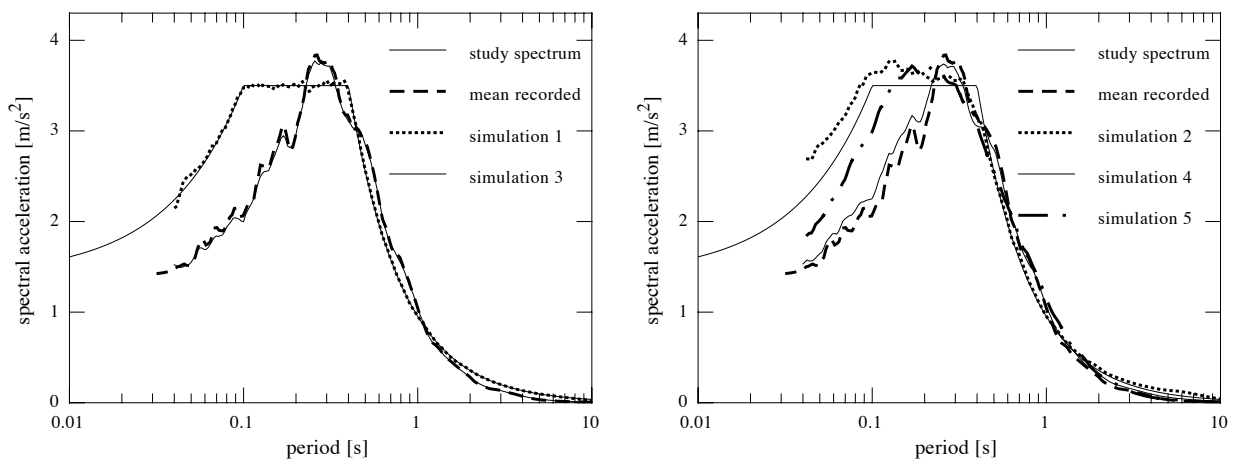

Figure 7: Average response spectra of the set of recordings and the five simulation options, over 100 time histories. The curves match perfectly the considered target spectrum (study spectrum or average of recordings) if iterations are used (simulation 1 and 3, left). Matching is also fulfilled by selection without iterations (simulation 2 and 4 , right). The simulation of Sabetta \& Pugliese (dotted line, right) allows a satisfactory matching of the study spectrum (dash dotted line) 
range. Five initial fundamental frequencies $(1.0,1.5,2.0,2.5$ and $3.0 \mathrm{~Hz})$ covering the range of frequencies of usual buildings are evaluated. Four different values of strength reduction factor are examined: $\mathrm{R}=2,3,4$ and 5. The following hysteretical models are used to compute the nonlinear responses: elastoplastic, $\gamma$-model, Q-model and the modified Takeda-model; the last two are also implemented with no stiffness reduction during the unloading phase. The viscous damping ratio $\xi$ is set to $5 \%$ throughout the study. The force-displacement relationships defining the six hysteretic models that are considered in the investigations are plotted in Figure 8 and described below:

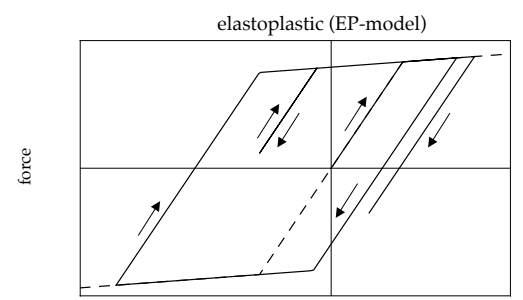

Q-model: $\alpha=0.0$

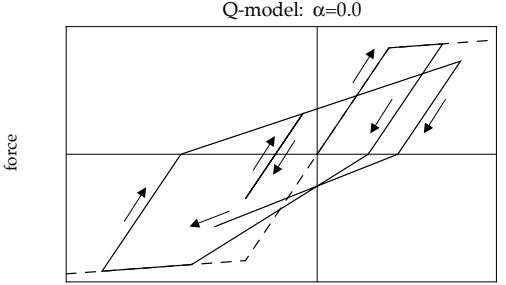

Q-model: $\alpha=0.4$

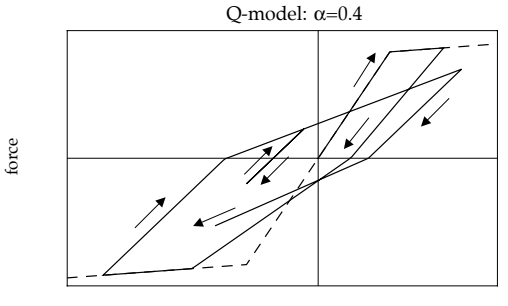

displacement

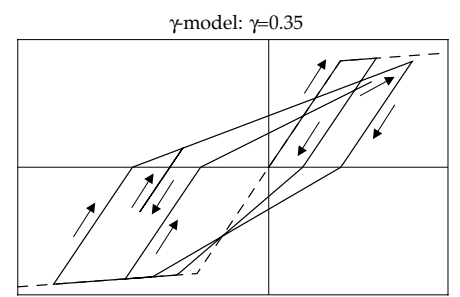

Takeda: $\alpha=0.0, \beta=0.0$
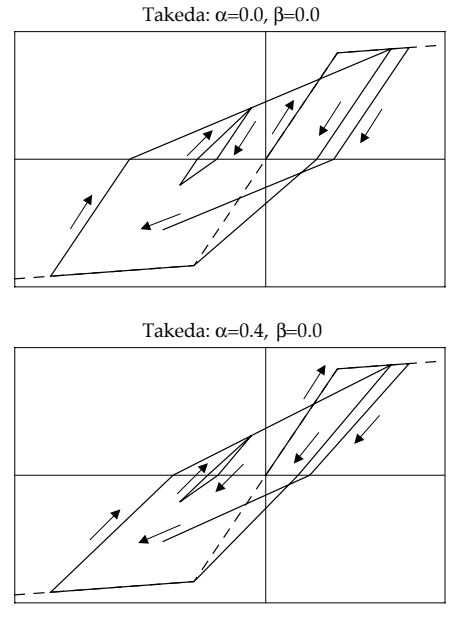

displacement

Figure 8: The six hysteretic models that were used in the investigations with non-linear SDOF.

1) Elastoplastic model: The elastoplastic model (EP-model) is sometimes also called bi-linear model. Even if it is mainly intended for elastoplastic material, such as steel, this model is extensively used for all types of materials because of its simplicity. The force-displacement relationships of the EP-model are specified using only three parameters: the stiffness, the yield displacement and the post yield stiffness expressed as a portion of the stiffness. The main drawback of the EP-model is the very stiff reloading curve after yielding and unloading. For instance, this characteristic does not take into account the closure of the cracks for the simulation of reinforced concrete. It leads to excessive energy dissipation by the inelastic cycles and to unrealistic permanent deformations.

2) Gamma-model: According to Lestuzzi et al. (2003), the EP-model is modified with a condition for the reloading curves specified by a supplementary parameter $\gamma$. For large yield excursions (displacements greater than current peak displacement), the reloading curves cross the elastic portion of the envelope at a height of $1-\gamma$ of the yield force. Otherwise (displacements smaller than current peak displacement), the reloading curves aim for the current peak displacement. The force-displacement relationships of the $\gamma$-model are specified through four parameters: the stiffness, the yield displacement, the post yield stiffness and $\gamma$. The value of $\gamma$ is determined empirically. Similar to the EP-model, the $\gamma$-model does not consider stiffness degradation due to increasing damage. 
3) Modified Takeda-model: Since the modified Takeda-model includes realistic conditions for the reloading curves, it provides a much better simulation of the features of material such as reinforced concrete in comparison to the EP-model. Moreover, the modified Takeda-model takes into account the degradation of the stiffness due to increasing damage, which is an important feature of reinforced concrete subjected to seismic loading (Saatcioglu, 1991). However, the modified Takeda-model does not include strength degradation. The Takeda-model was initially proposed in an original version by Takeda et al. (1970). The modified Takedamodel was developed independently by Otani (1974) and Litton (1975). It was adapted afterwards by many authors. The version used here is the one of Allahabadi and Powell (1988). The force-displacement relationships of the modified Takeda-model are specified through five parameters: the initial stiffness, the yield displacement, the post yield stiffness, a parameter $(\alpha)$ relating the stiffness degradation and another $(\beta)$ specifying the target for the reloading curve. Different rules are used for large and for small hysteretic cycles. The small cycles are further divided into small cycles with yielding and small cycles with small amplitudes.

4) Modified Takeda-model (zero): This model considers a specific and simple case of modified Takeda-model where $\alpha=0$ i.e. no degradation of the unloading stiffness is taken into account.

5) Q-model: A simplified version of the modified Takeda-model was proposed by Saiidi and Sozen (1981), the Q-model. In comparison with the modified Takeda-model, the consideration of the absolute value of peak displacement for both directions constitutes the main simplification. Moreover, there are no distinctions between large and small hysteretic cycles. The reloading curves systematically target the point corresponding to the absolute value of actual peak displacement. Similar to the modified Takeda-model, the Q-model takes into account the stiffness degradation, but does not take into account strength degradation. The force-displacement relationships of the Q-model are completely specified using four parameters: the initial stiffness, the yield displacement, the post yield stiffness and a parameter $(\alpha)$ relating the stiffness degradation.

6) Q-model (zero): This model assumes that the factor $\alpha=0$. Hence, it does not consider the degradation of the unloading stiffness.

Since the sole effect of the seismic input is under focus in this study, it is necessary to reduce the number of other parameters influencing the structural response. This is the reason for setting the maximum elastic displacement of the examined SDOF equal to the target spectral displacement reached at the SDOF's frequency for all studied earthquakes (recorded and synthetic). Therefore, the yield displacements are the same while the $\mathrm{R}$ values turn out to be slightly different. By varying the frequency, the strength reduction factor and the hysteretic model, a single ground motion leads to $120(5 \times 4 \times 6=120)$ different dynamic non-linear responses.

\subsection{Results with SDOF}

First, it is pointed out that since the differences that arise when examining the influence of the hysteretic models are found to be not significant except for the EP-model, it was decided to aggregate the results yielded by the five other models. The non-linear response of the reference set of recorded earthquakes for a given $\left(f_{0}-R\right)$ couple is characterized by the average and standard deviation of the 9 earthquakes $\times 6$ hysteretic models $=54$ values. Because each simulation option is made of 100 events, 500 values determine the average and standard deviation in the case of synthetic earthquakes. Both the displacement and the energetic criterion are used to represent the dynamic non-linear response. 


\subsubsection{Ductility demand}

The definition used is that of the maximum non-linear displacement to the yield displacement ratio, which is namely the displacement ductility demand $\left(\mu_{\Delta}\right)$. Since constant yield displacements are used for the definition of the non-linear SDOF, the results of displacement demand correspond to that of displacement ductility demand. Typical results are illustrated in Figure 9.
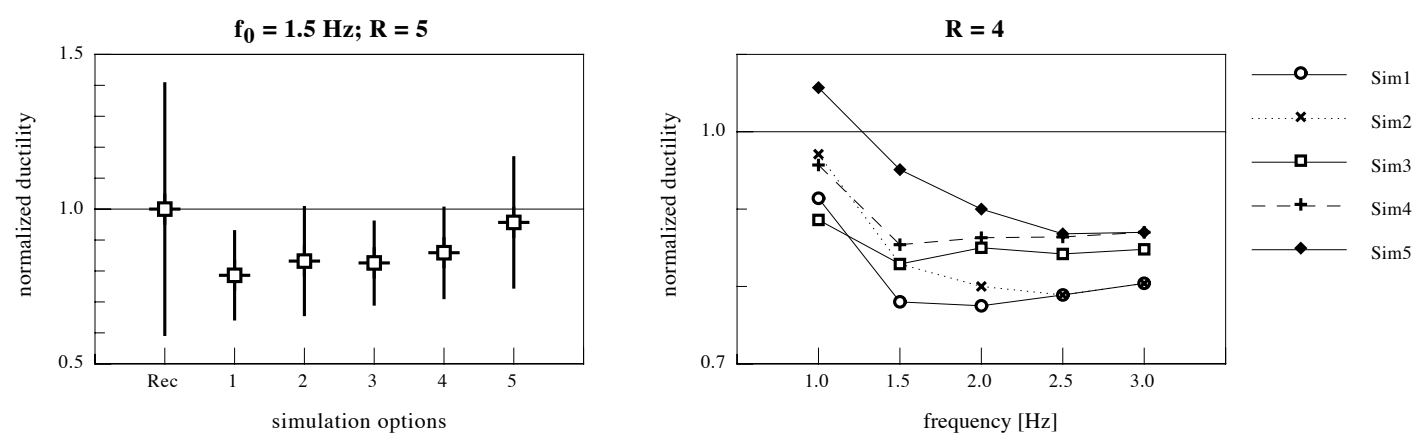

Figure 9: Mean values (square marks) and standard deviations (thin vertical lines) of the normalized (by the values corresponding to the recorded events) displacement ductility demands (left). Mean values of the normalized displacement ductility demands as a function of the frequency (right).

The following general comments can be made on the basis of the results:

- The use of synthetic earthquakes generally leads to an underestimation of the ductility demand.

- Simulation option 5 (Sabetta \& Pugliese) best reproduces the features (mean values) observed for recorded earthquakes.

- When the study spectrum is used to generate earthquakes, iterations (simulation option 1) should be avoided since the extra computation effort does not improve the order of magnitude of the ductility demand (simulation option 2). In fact, the standard deviation drops. The results for simulation option 1 (original SIMQKE) are unable to reach the average for the recorded earthquakes, even when the standard deviation is considered.

- Simulation option 4 (SIMQKE, average recorded as target, without iterations) is the best among the stationary ones.

- The large difference between the standard deviation obtained for the recorded ground motions and that for the simulations is mainly due to the fact that the hundred corresponding synthetic earthquakes are related to a smooth average response spectrum. Consequently, their individual variability about their target spectrum is less than that of the nine records.

- The right hand side of Figure 9 shows that the accuracy with which the simulations reproduce the ductility demand worsens for higher frequencies. The same figure also confirms that simulation option 5 (Sabetta \& Pugliese) is the best simulation option for all frequencies.

- The trend among the simulations remains the same irrespective of the value of the strength reduction factor.

\subsubsection{Energy demand}

The input energy is equal to the integral over the relative displacement of the three resisting forces, namely: "inertia", damping and elastic. The hysteretic energy, which is the amount of 
energy the structure has to dissipate by plastic deformations, corresponds to the area delimited by the non-linear force displacement graph. The study of the input and hysteretic energy confirms and stresses the findings drawn from the displacement ductility demand analysis. Typical results are illustrated in Figure 10 for the hysteretic energy.
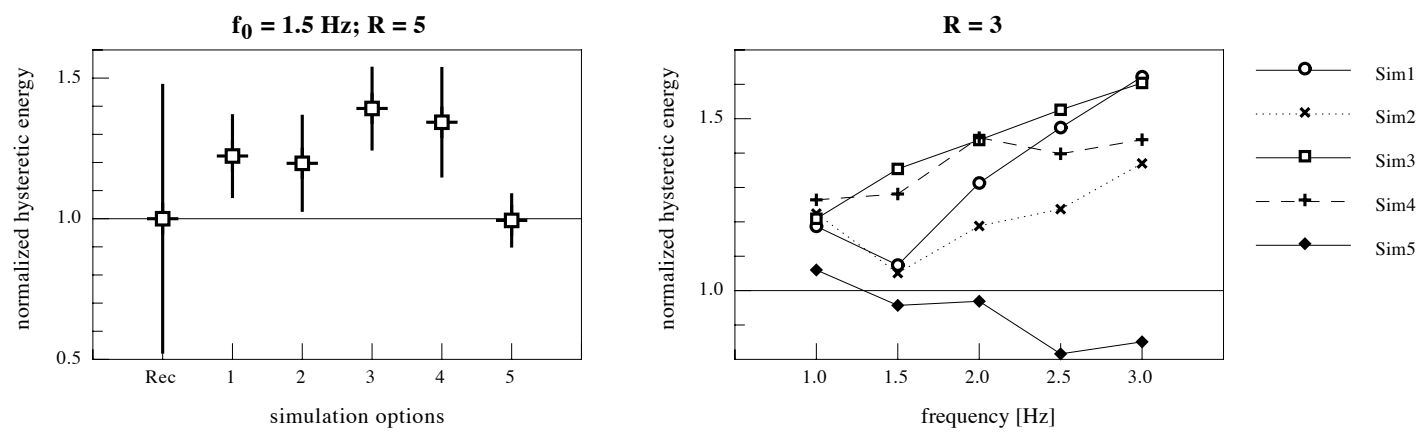

Figure 10: Mean values (square marks) and standard deviations (thin vertical lines) of the normalized (by the values corresponding to nine recorded events) hysteretic energies (left). Mean values of the normalized hysteretic energies as a function of the frequency (right).

The following general comments applies to the results:

- Stationary synthetic earthquakes and specifically those targeting the study spectrum significantly overestimate the energetic values.

- As for ductility demand, simulation option 5 (Sabetta \& Pugliese) gives the best overall results. However, it is not conservative.

- Iterations worsen both the order of magnitude and the standard deviation (simulation option 1 vs 2 and simulation option 3 vs 4 ).

- The overestimation of the hysteretic energy by stationary simulation is significant for high frequencies. No such steady trend can be pointed out regarding simulation option 5 (Sabetta \& Pugliese).

- The value of the strength reduction factor $\mathrm{R}$ has no impact on the trend among the simulation options.

- The accuracy with which the simulation options reproduce the structural responses is not dependent on the consideration of the input or hysteretic energy.

\section{Investigations with MDOF}

For SDOF, it is evident from the results that non-stationary simulations provide much better correspondence with recorded earthquakes than stationary simulations for both the displacement ductility demand and hysteretic energy. To verify if these preliminary conclusions are also valid for real multiple story structural wall buildings, a second investigation is performed with MDOF. The objective is to conduct the computations in the same manner as a design engineer. This means that only a few time histories, instead of hundred, are used to catch the variability of the non-linear response. 


\subsection{Characteristics of the examined shear wall buildings}

Figure 11 shows as example, the four-story reinforced concrete shear wall buildings examined in the investigations with MDOF. These multiple story buildings feature a typical structural wall system consisting of flat slabs, small columns designed for only gravity loads and two relatively slender structural walls of rectangular cross section in each directions. Such structural wall systems are popular in regions of central Europe, such as in Switzerland. On the whole ten buildings are examined. The characteristics of the buildings and the characteristics of their reinforced concrete shear walls are given in Table 3. These characteristics (number of stories, story height, wall dimensions, story mass, axial load and reinforcement) are chosen in order to feature the same frequencies as the studied SDOF while keeping the related values within realistic ranges.

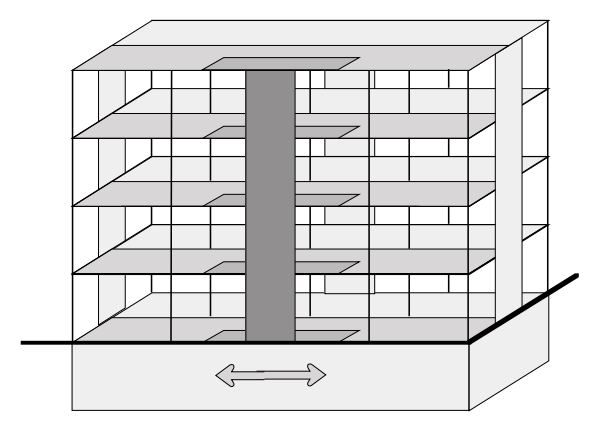

Figure 11: Sketch for the four-story reinforced concrete shear wall buildings used in MDOF investigations.

The walls are designed according to the capacity design method using the equivalent force method. It is necessary to impose high values of $\mathrm{R}$ to ensure significant non-linear excursions. The non-linear seismic response is computed using the dynamic non-linear finite element program IDARC (Valles et al., 1996). The moment curvature envelope used for the trilinear hysteretic model is computed by means of a fibre model, which requires the building's and wall's geometry (Table 3 ) as well as their material properties (see Table 3 for reinforcement yield strength $f_{y}$ and reinforcement ratios $\rho_{\text {edge }}$ and $\rho_{\text {center }}$ ). The last row of Table 3 gives the corresponding computed flexural strength ( $\left.\mathrm{M}_{\text {IDARC }}\right)$.

Table 3: Properties of the multiple story buildings and their reinforced concrete shear walls for MDOF investigations.

\begin{tabular}{|c|c|c|c|c|c|c|c|c|c|c|c|}
\hline \multicolumn{2}{|c|}{ Number of stories } & 4 & 4 & 4 & 4 & 4 & 4 & 3 & 3 & 3 & 3 \\
\hline \multicolumn{2}{|c|}{ strength reduct. fact. R } & 6 & 6 & 6 & 6 & 4 & 4 & 6 & 6 & 6 & 6 \\
\hline \multicolumn{2}{|c|}{ fund. frequency $\mathrm{f}_{0}[\mathrm{~Hz}]$} & 1 & 1.5 & 2 & 2.5 & 2 & 2.5 & 1 & 1.5 & 2 & 2.5 \\
\hline story height & {$[\mathrm{m}]$} & 2.6 & 2.6 & 2.6 & 2.6 & 3 & 3 & 3.2 & 3 & 3 & 3 \\
\hline wall length & {$[\mathrm{m}]$} & 3.3 & 3.5 & 3.5 & 4 & 4.2 & 4.2 & 3.2 & 3.8 & 3.8 & 3.8 \\
\hline wall depth & {$[\mathrm{m}]$} & 0.4 & 0.4 & 0.4 & 0.4 & 0.3 & 0.3 & 0.3 & 0.3 & 0.3 & 0.3 \\
\hline story mass & {$[\mathrm{t}]$} & 900 & 485 & 273 & 260 & 224 & 147 & 760 & 840 & 470 & 300 \\
\hline axial load & {$[\mathrm{KN}]$} & 1940 & 1960 & 1960 & 2020 & 1980 & 1980 & 1420 & 1460 & 1460 & 1460 \\
\hline $\mathrm{f}_{\mathrm{y}}$ & {$[\mathrm{MPa}]$} & 400 & 400 & 300 & 300 & 400 & 400 & 460 & 460 & 460 & 460 \\
\hline$\rho_{\text {edge }}$ & {$[\%]$} & 1.9 & 1.3 & 1.0 & 1.0 & 1.3 & 0.3 & 1 & 2.1 & 1.3 & 0.5 \\
\hline$\rho_{\text {center }}$ & {$[\%]$} & 0.2 & 0.1 & 0.1 & 0.1 & 0.2 & 0.2 & 0.2 & 0.3 & 0.2 & 0.2 \\
\hline $\mathrm{M}_{\text {IDARC }}$ & {$[\mathrm{MNm}]$} & 4.6 & 4.5 & 3.9 & 4.8 & 5.4 & 4.9 & 2.8 & 5.1 & 4.3 & 3.7 \\
\hline
\end{tabular}




\subsection{Results}

In order to compare the results corresponding to the nine recorded earthquakes, each simulation option uses nine synthetic earthquakes randomly picked among the hundred time histories generated for the SDOF study. It is also verified whether the results remain the same if other groups of nine earthquakes are examined. A unique hysteretic (trilinear) model is implemented for the study. The displacement on the top floor is chosen to characterize the nonlinear behavior. Due to the influences of higher modes, it is difficult to unequivocally define a yield displacement that could be used to compute the displacement ductility demand.

This study on realistic structures confirms the conclusions derived from the study on SDOF. Typical results are presented in Figure 12.

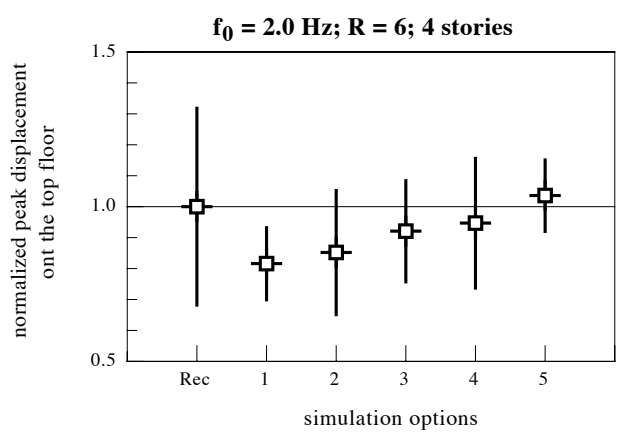

Figure 12: Mean values (square marks) and standard deviations (thin vertical lines) of the normalized (by the values corresponding to the recorded events) peak displacement on the top floor of a 4 story building.

The following general comments applies to the results:

- Synthetic earthquakes generally underestimate the displacement on the top floor.

- Iterations significantly worsen the variability. Fearing that using no iterations may produce a too big standard deviation is not justified.

- The mean value trends are similar to those observed for SDOF. However, the average difference from the set of recorded earthquakes is significantly less for MDOF. This especially applies for simulation options 3 and 4 (SIMQKE, average recorded as target).

- Similar to the results with SDOF (see 3.2), the best and the worst simulation is simulation option 5 (Sabetta \& Pugliese) and simulation option 1 (classical SIMQKE with iterations) respectively.

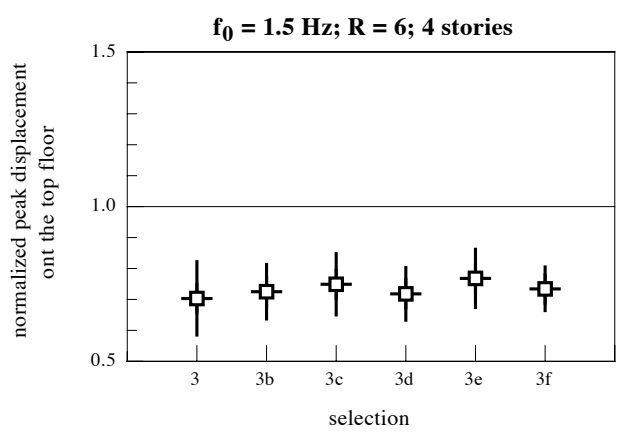

Figure 13: Six runs of simulation option 3 for six different groups of nine synthetic earthquakes picked among the hundred generated ground motions showing that the results are practically not sensitive to the chosen time histories. 
As Figure 13 shows, the trend displayed here and upon which the conclusions are based, remain the same if the nine examined events are chosen otherwise among the 100 generated time histories.

\section{Conclusions}

The investigations reported in this paper compare two different methods for generating synthetic earthquakes: the classical stationary simulation of SIMQKE (with five various options) and the non-stationary simulation of Sabetta \& Pugliese. The comparison is focused on the accuracy of the reproduction of the statistical characteristics of the structural non-linear response of ductile wall systems due to recorded earthquakes. The results obtained with non-linear SDOF, defined by six recognized hysteretic models, show that the used simulation considerably influence the non-linear seismic behavior. For both displacement ductility demand and energy demand, the non-stationary simulation gives significantly better results than stationary simulation, which always leads to a strong underestimation of the ductility demand and a strong overestimation of the energy demand. Among the different investigated options for the stationary simulations, the classical method of using an iterative process for matching the target spectrum produces the worst results for both mean values and standard deviation. As an important consequence of these results and by contrast to a common accepted assumption, it may be asserted that, regarding displacement demand, synthetic time histories covering the whole frequency range of a design spectrum definitely do not represent a conservative input for nonlinear systems. Finally, the results obtained for realistic multiple story buildings with non-linear MDOF confirm the conclusions with SDOF. However, the average difference from the set of recorded earthquakes is less for MDOF.

In brief, the following design implications should be retained:

- Non-stationary simulation should be preferred to stationary simulations

- When using stationary simulation, do not use the procedures for matching the target spectrum

It should be noted that the above conclusions are related to the used yield displacements. To focus the investigations on the earthquakes only, the yield displacements are the same while the $\mathrm{R}$ values turn out to be slightly different. Since constant yield displacements are used, the results of displacement demand correspond to that of displacement ductility demand.

It should be further noted that the above conclusions are only valid for ductile wall systems, i.e. wall systems such as capacity designed slender reinforced concrete structural walls that develop a flexural and not a shear driven failure mechanism. This study did not amount for the impact of strength degradation. As a consequence, materials such as unreinforced masonry may exhibit different behavior as the strength degradation definitely needs to be taken into account. In this case, additional parameters like strong motion duration, for instance, may arise.

Ultimately, it should be stressed that only one set of recorded earthquakes, featuring a specific response spectra, was used. It would be therefore of interest to enlarge the investigations by changing the set of recorded events, and thus the target spectrum. The conclusions made so far could also be enhanced by increasing the number of studied frequencies in the range of realistic building frequencies. 


\section{References}

Allahabadi R. and Powell G. H. (1988) Drain-2DX User Guide. Report No. UCB/EERC-88/06. College of Engineering, University of California, Berkeley.

Elenas A. (2002) Seismic Damage Potential Described by Spectral Intensity Parameters. Procceedings of the 12th European Conference on Earthquake Engineering, Paper 267, London.

Eurocode 8 (2004). Design of Structures for Earthquake Resistance, Part 1: General Rules, Seimic Actions and Rules for Buildings, EN 1998-1; 2004; European Committee for Standardization (CEN), Brüssel.

Federal Emergency Management Agency (FEMA 273). (1997) NEHRP Guidelines for the Seismic Rehabilitation of Buildings, Washington D.C.

Gasparini D. A. and Vanmarcke E. H. (1976) Simulated earthquake motions compatible with prescribed response spectra. MIT Civil Engineering Research Report R76-4. Massachusetts Institute of Technology, Cambridge, Mass.

Lestuzzi P. and Badoux M. (2003) The gamma-Model: A Simple Hysteretic Model For Reinforced Concrete Walls. Paper $n^{\circ}$ 126. Proceedings of the fib-Symposium: Concrete Structures in Seismic Regions, Athens, Greece.

Lestuzzi P., Schwab P., Koller M. and Lacave C. (2004) How to Choose Earthquake Recordings for Non-linear Seismic Analysis of Structures. Proceedings of 13th World Conference on Earthquake Engineering, Vancouver, British Columbia, Canada.

Litton R. W. (1975) A contribution to the analysis of concrete structures under cyclic loading. Ph.D. Thesis, Civil Engineering Dept., University of California, Berkeley.

Nassar A.A. and Krawinkler H. (1991) Seismic Demands for SDOF and MDOF Systems. Research Report No. 95, The John A. Blume Earthquake Engineering Center, Department of Civil Engineering, Stanford University, Stanford, California.

Otani A. (1974) Inelastic analysis of R/C frame structures. J. Struct. Div., ASCE 100 ST/, 14331449.

Saactioglu M. (1991) Modeling hysteretic force deformation relationships for R/C elements. Earthquake-Resistant Concrete Structures.: Inelastic Response and Design. Special publication SP-127 of the American Concrete Institute (ACI). Detroit, Michigan.

Sabetta F. and Pugliese A. (1996) Estimation of Response Spectra and Simulation of Nonstationary Earthquake Ground Motions. Bulletin of the Seismological Society of America, Vol 86, No. 2, pp. 337-352.

Saiidi M. and Sozen M. A. (1981) Simple nonlinear seismic analysis of R/C structures. Journal of the Structural Division. Proceedings of the American Society of Civil Engineers (ASCE). Vol. 107, No. ST5, 937-952.

Schwab P. (2002) Influence of seismic characteristics on the dynamic nonlinear response of structures. Master thesis, Ecole Polytechnique Fédérale de Lausanne (EPFL), Switzerland.

Smit P. et al. (2000) European Strong Motion Database. European Council, Environment and Climate Research Programme, CD-ROM.

Takeda T., Sozen M. A. and Nielsen N. N. (1970) Reinforced concrete response to simulated earthquakes. Journal of the Structural Division. Proceedings of the American Society of Civil Engineers (ASCE). Vol. 96, No. ST12, 2557-2573.

Valles R. E., Reinhorn A. M., Kunnath S. K., Li C. and Madan A. (1996) IDARC 2D Version 4.0: A Program for Inelastic Damage Analysis of Buildings. Technical Report NCEER-96-0010. State University of New York at Buffalo, NY. 